\title{
Lipid/PLGA hybrid microbubbles as a versatile platform for noninvasive image-guided targeted drug delivery
}

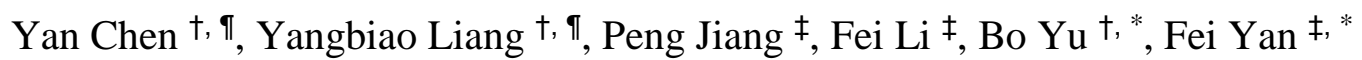

† Ultrasound Medical Center, Zhujiang Hospital of Southern Medical University, Guangzhou, 510282, China

‡ Paul C. Lauterbur Research Center for Biomedical Imaging, Institute of biomedical and Health Engineering, Shenzhen Institutes of Advanced Technology, Chinese Academy of Sciences, Shenzhen, 518055, China

*Correspondence to: Dr. Bo Yu. Zhujiang Hospital of Southern Medical University, 253 Industrial Avenue, Guangzhou, China 510282. Email address: gzyubo@163.com. Dr. Fei Yan. Shenzhen Institutes of Advance Technology, Chinese Academy of Science, 1068 Xueyuan Avenue, Shenzhen University Town, Shenzhen, China 518055. Email address: fei.yan@ siat.ac.cn.

I These two authors equally contributed to this work. 
(a)

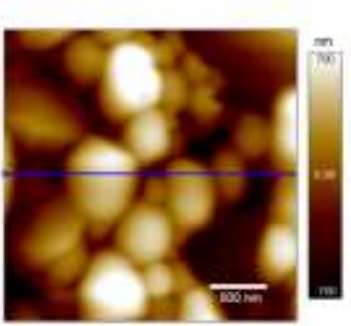

(b)

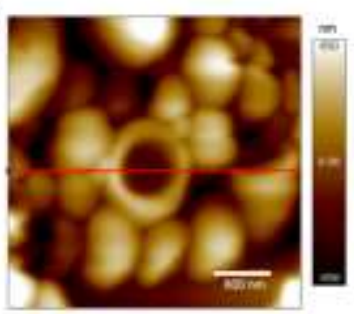

(c)

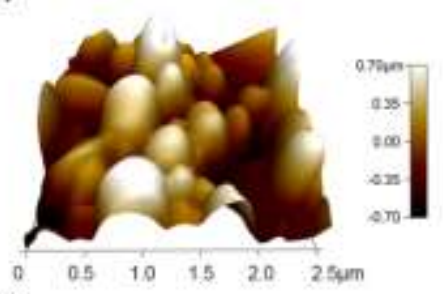

(d)

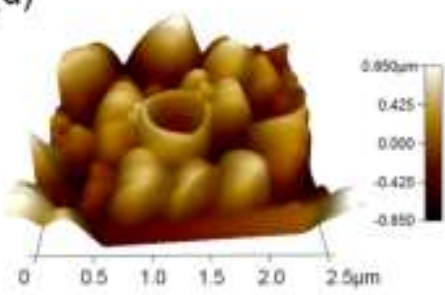

(e)

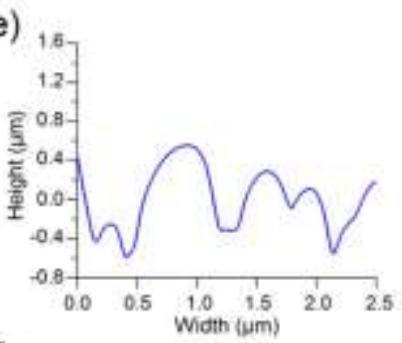

(f)

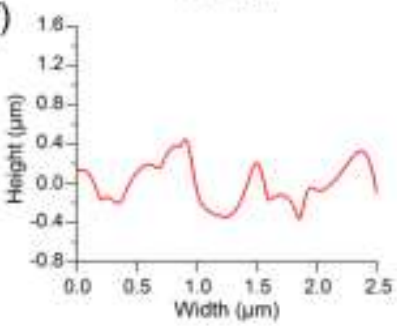

Figure S1. (a) and (b) Topographical view obtained by AFM analysis shows the morphology of the PLGA MBs and lipid/PLGA MBs, respectively. (c) and (d) 3D reconstruction image of PLGA MBs and lipid/PLGA MBs according to the above data. (e) and (f) Height-graphs derived from the corresponding images.

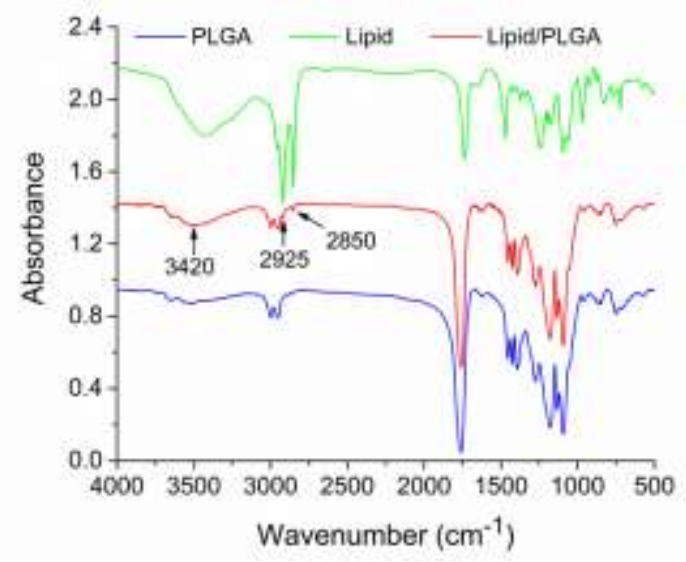

Figure S2. FTIR spectral analysis of PLGA MBs, lipid MBs and lipid/PLGA MBs. 

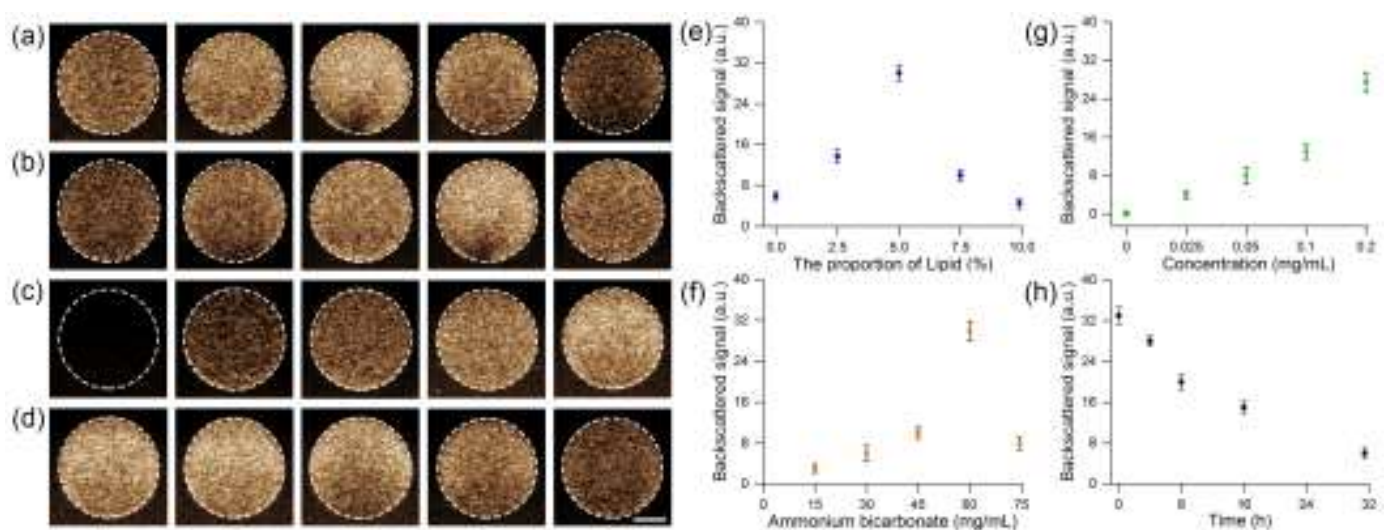

Figure S3. (a) The contrast imaging ability of lipid/PLGA MBs when the concentration of $\mathrm{NH}_{4} \mathrm{HCO}_{3}$ was $60 \mathrm{mg} / \mathrm{mL}$ and the proportion of lipid in PLGA was set at $0,2.5,5.0,7.5$, and $10.0 \%(\mathrm{w} / \mathrm{w})$ (images from left to right). (b) The contrast imaging ability of lipid/PLGA MBs when the ratio of lipid was 5\% and the concentration of ammonium bicarbonate was set at 15, 30, 45, 60, and $75 \mathrm{mg} / \mathrm{mL}$, respectively (images from left to right). (c) Ultrasound images of a gel phantom containing lipid/PLGA MBs at different concentrations which were $0,0.025,0.05,0.1$, and $0.2 \mathrm{mg} / \mathrm{mL}$ (images from left to right). (d) In vitro stable contrast enhanced ultrasound imaging analysis. The echo intensity of lipid/PLGA MBs changed over time from $0,4,8,16$, and $32 \mathrm{~h}$ (images from left to right). Scale bar $=4 \mathrm{~mm}$. (e)-(h) Corresponding average backscattered signal value obtained from ultrasonic images in (a)-(d) $(\mathrm{N}=5)$. 


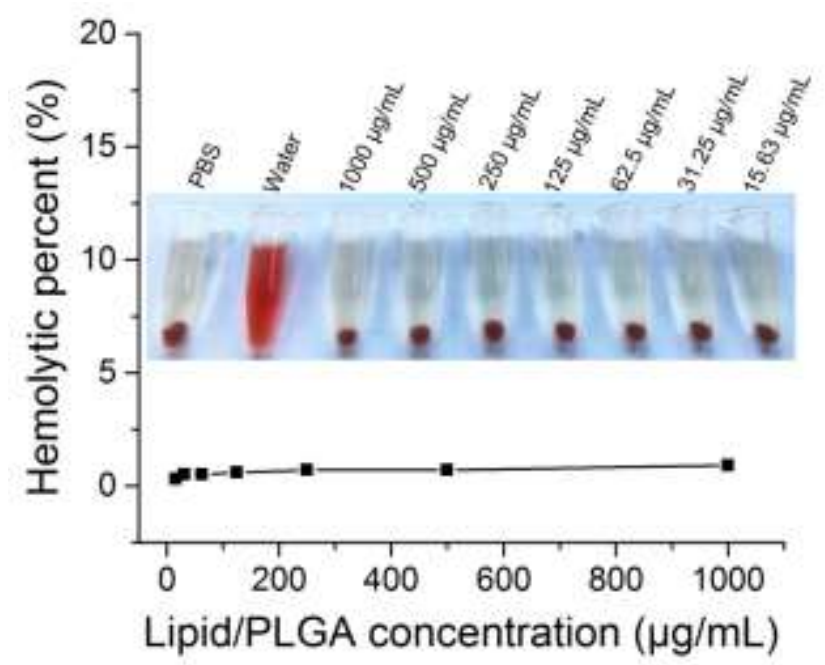

Figure S4. Hemolysis rate of lipid/PLGA MBs at different concentrations (15.63, $31.25,62.5,125,250,500,1000 \mu \mathrm{g} / \mathrm{mL})$.

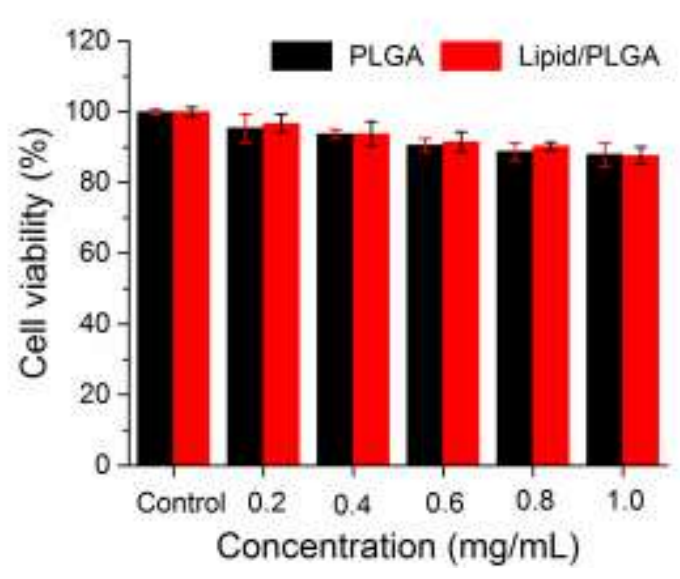

Figure S5. Cell viabilities of $4 \mathrm{~T} 1$ cells after the co-incubation with PLGA MBs or lipid/PLGA MBs at the same concentrations (0.2, 0.4, 0.6, 0.8, $1.0 \mathrm{mg} / \mathrm{mL})$, respectively $(\mathrm{N}=5)$. 


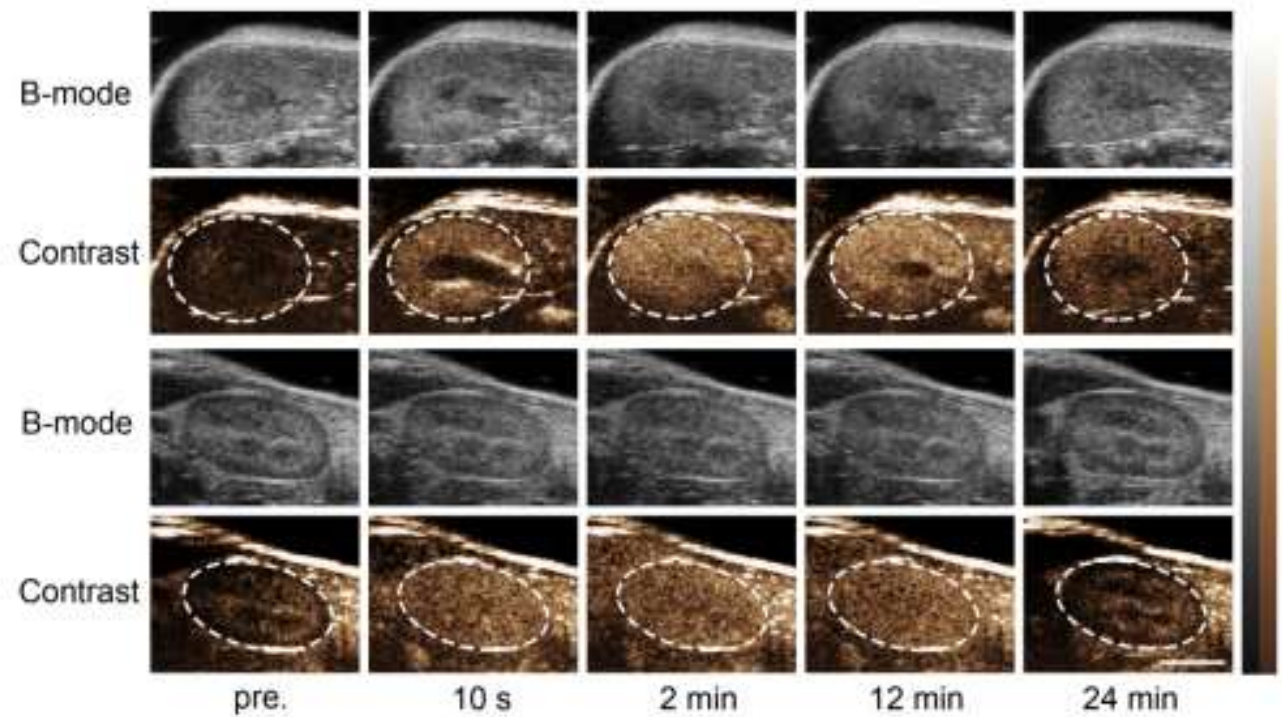

Figure S6. Typical spleen (the upper two rows) and kidney (the lower two rows) ultrasound images after administration of lipid/PLGA MBs. The white dotted line circles denote the organ area. Scale bar $=5 \mathrm{~mm}$.

(a)

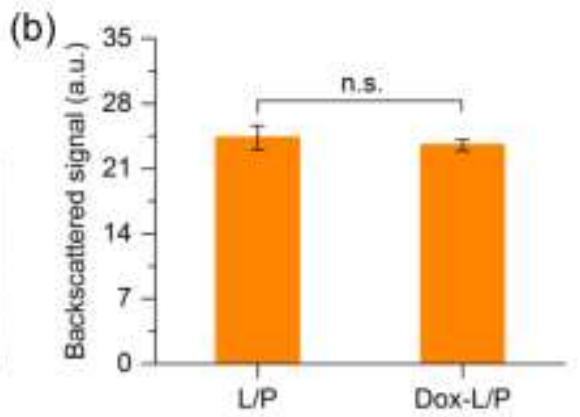

Figure S7. (a) Ultrasound image comparison between lipid/PLGA MBs and Dox-lipid/PLGA MBs in PBS. (b) Histogram derived from the corresponding ultrasound image analysis. Mean intensity reported for all samples are representative of three investigations $(\mathrm{N}=5)$. 


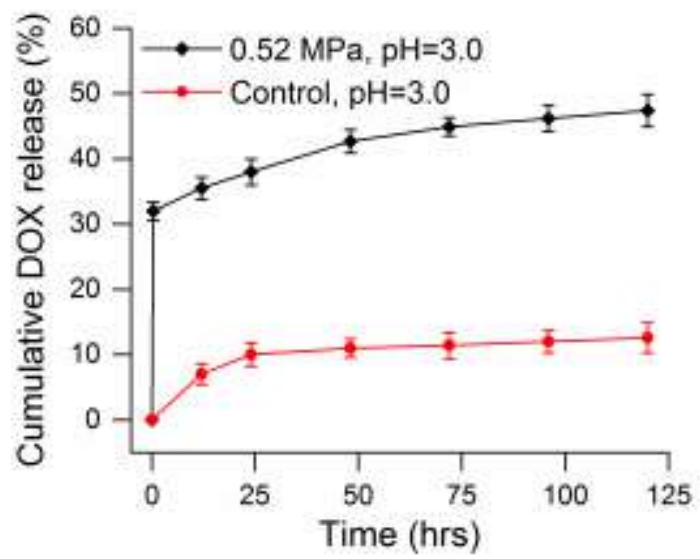

Figure S8. Release of Dox from Dox-lipid/PLGA MBs after treatment with ultrasound for $1 \mathrm{~min}$ at $0.52 \mathrm{MPa}$ or without ultrasound under acidic buffer solutions $(\mathrm{pH}=3.0)$.

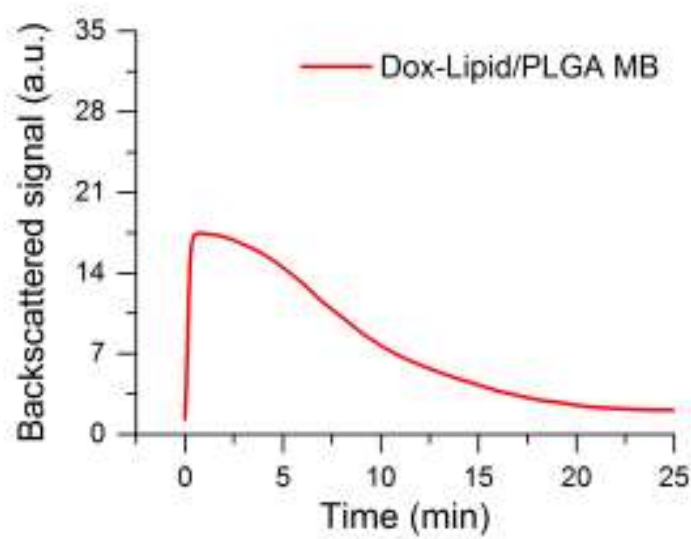

Figure S9. The corresponding quantitative analysis of the ultrasound imaging signal intensity from Dox-lipid/PLGA MBs treated group was shown.

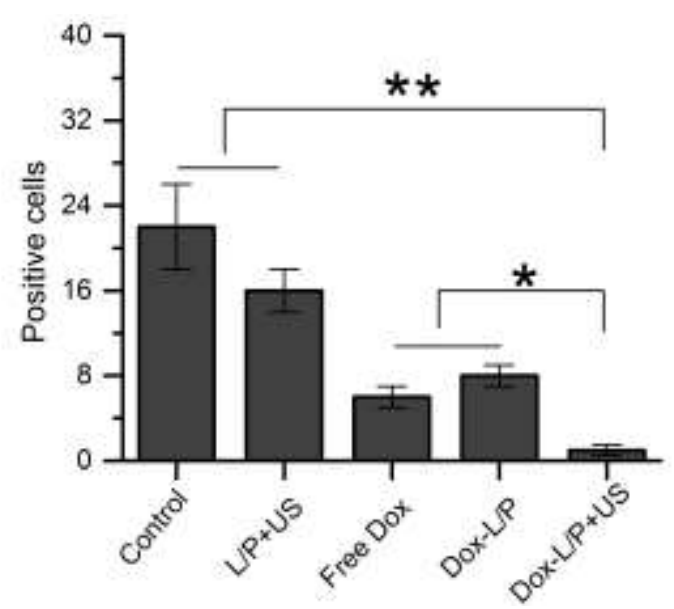

Figure S10. The number of positive cells per group in Ki-67 assay $(\mathrm{N}=5)$. 


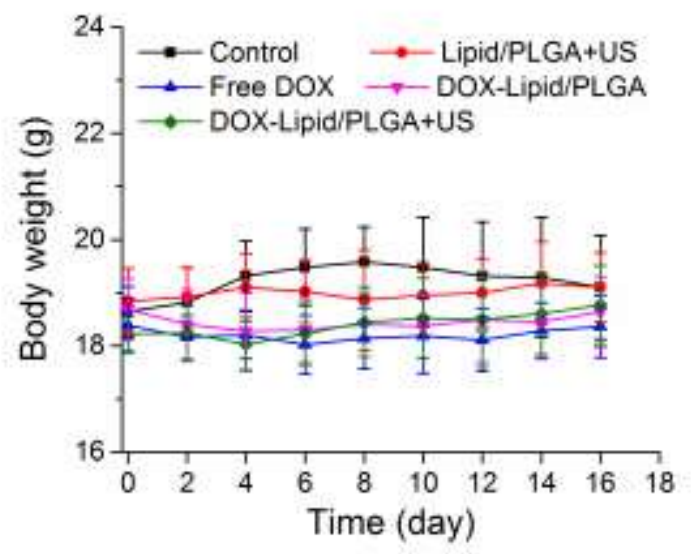

Figure S11. Body weight curves for different groups during the experiments.

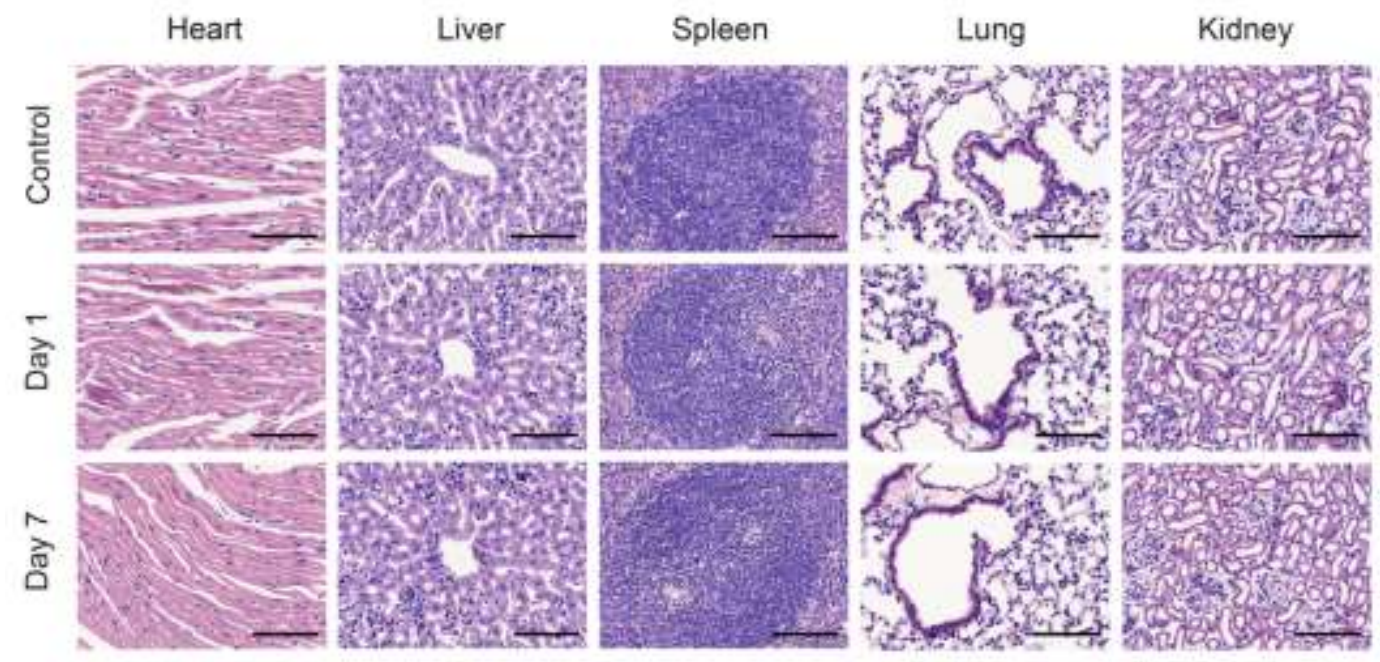

Figure S12. H\&E histological staining examinations of major organs (heart, liver, spleen, lung and kidney) of saline and intravenous-injection groups at a dose of 5 mg/kg (Dox-lipid/PLGA MBs) after 1 and 7 day. Scale bar $=100 \mu \mathrm{m}$. 